poxämischen COPD-Patienten klar darlegen, dass kapilläre $\mathrm{PaO}_{2}$-Werte zu tief gemessen werden, was zu einer Überversorgung mittels LTOT in $21 \%\left(\mathrm{PaO}_{2}<55 \mathrm{mmHg}\right.$ als Cut-Off $)$ sowie $30 \%\left(\mathrm{PaO}_{2}<60 \mathrm{mmHg}\right.$ als Cut-off $)$ geführt hätte [7].

Sicherlich müssen Leitlinien auch die Anwendbarkeit und klinische Machbarkeit der empfohlenen Maßnahmen berücksichtigen. Vor dem Hintergrund der Konsequenzen sowohl für den Patienten als auch die hiermit verbundenen therapiebezogenen Kosten ist es aber essenziell, diesen und auch die anderen genannten Punkte im Rahmen einer Revision der Leitlinie komplett neu zu bewerten.

Weiter bleibt herauszustellen, dass die Evidenz für die LTOT bei der COPD aus Studien der späten 1970er Jahre stammt. Somit bleibt fraglich, inwieweit Verbesserungen in Diagnostik und Therapie der COPD und auch ihrer Komorbiditäten dazu beigetragen haben, dass eine Überlebensverbesserung durch LTOT heutzutage tatsächlich weniger relevant geworden ist. Gleichwohl dies im Bereich des Spekulativen bleibt, kann heute dennoch festgestellt werden, dass eine zu großzügige Verschreibung von Sauerstoff kritisch hinterfragt werden muss.

An dieser Stelle muss betont werden, dass die DGP-Leitlinien zur LTOT dringend überarbeitet werden müssen, um eine Überversorgung mit Sauerstoff zu vermeiden. Dies betrifft auch die vorschnelle Verordnung einer LTOT bei passagerer Hypoxämie im Rahmen einer Exazerbation, wie es in Deutschland gelebte Praxis ist.

\section{Außerklinische Beatmung}

Im Gegensatz zur LTOT liegen gegenwärtig aktuell revidierte Leitlinien der DGP zur außerklinischen Beatmung vor [2]. Diese Leitlinie beschäftigt sich in vollem Umfang mit vielen Aspekten der außerklinischen Beatmung bei einer Vielzahl von zugrunde liegenden Entitäten und Erkrankungen. Dabei haben auch hier in Bezug auf die COPD aktuelle Studiendaten in der Tat dazu beigetragen, den Therapiealgorithmus für die außerklinische Beatmung mittels NIV zu verändern. Das Beispiel zeigt, wie wesentlich die Revision von Leitlinien ist, wenn neue Studiendaten vorliegen.

Mittlerweile ist es auch international anerkannter Konsens, dass eine Langzeit-NIV bei chronisch hyperkapnischer COPD sinnvoll ist und die Prognose der Patienten verbessern kann. Dabei zeichnet die aktuelle Studienlage mittlerweile ein sehr detailliertes Bild davon, wann eine Langzeit-NIV indiziert ist und wann nicht. So muss heute eine fatalistisch-negative Haltung, die eine Langzeit-NIV dem Patienten vorenthält, ebenso vermieden werden wie eine euphorisch-positive Haltung, die gegebenenfalls zu einer Überversorgung führt. Dies betrifft sowohl die Einleitung als auch die Kontrolle der außerklinischen Beatmung und tangiert auch das sensible Thema der Ökonomie.

So konnte zunächst gezeigt werden, dass Patienten mit stabiler hyperkapnischer COPD eine klare Prognoseverbesserung erfahren. Im Gegensatz dazu war die Frage nach der Einleitung einer Langzeit-NIV in der Folge einer Akut-Beatmung in der Klinik noch zweifelhaft gewesen. Eine holländische Studie konnte 2014 zeigen, dass eine unselektionierte Fortführung der NIV in der Folge einer Akut-Beatmung in der Klinik keine Vorteile hinsichtlich Prognose und Re-Hospitalisierung bietet [8]. So konnte auch eine aktuelle Studie zur Kontrolle der NIV zeigen, dass bei einem signifikanten Teil der Patienten die Fortset- zung einer NIV nicht notwendig und nicht gerechtfertigt war, insbesondere wenn bei Patienten in der Folge einer Akut-NIV in der Klinik eine Langzeitbeatmung eingeleitet wurde [9].

Im Unterschied zur holländischen Studie wurde in einer aktuellen britischen Studie eine Langzeit-NIV erst dann eingeleitet, wenn eine Hyperkapnie über mindestens 2 Wochen persistiert hatte [10]. Für diesen Fall konnte bei diesen schwerstkranken Patienten der kombinierte Endpunktes aus Re-Hospitalisierung und Tod ebenfalls deutlich gesenkt werden.

Die differenzierte Studienlage macht klar, dass gerade bei $\mathrm{Pa}-$ tienten nach schwerer hospitalisationspflichtiger Akut-Exazerbation darauf geachtet werden muss, eine Überversorgung einerseits zu vermeiden, andererseits aber keine Unterversorgung zu riskieren. Aufgrund der immer kürzeren stationären Behandlungszeiten macht dies eine intersektorale Zusammenarbeit zwischen stationärer und ambulanter Krankenversorgung notwendig. Auch dieses Beispiel zeigt, dass wissenschaftlich begründete Evidenz, zusammengefasst und in Form von Empfehlungen formuliert, wichtig ist, aber auch die Versorgungsstruktur und die Versorgungslandschaft in Deutschland so verändert werden muss, dass eine optimale Umsetzung von Evidenz möglich ist.

Literatur

1. Magnussen H, Goeckenjan G, Koehler D et al. Leitlinien zur Langzeit-Sauerstofftherapie. Pneumologie. 2001;55:454-64.

2. Windisch W, Dreher M, Geiseler J et al. für die Leitliniengruppe "Nichtinvasive und invasive Beatmung als Therapie der chronischen respiratorischen Insuffizienz". S2k - Leitlinie: Nichtinvasive und invasive Beatmung als Therapie der chronischen respiratorischen Insuffizienz - Revision 2017. Pneumologie 2017; 71:722-95.

3. Long-Term Oxygen Treatment Trial Research G. A randomized trial of long-term oxygen for COPD with moderate desaturation. N Engl J Med. 2016;375:1617-27.

4. Magnussen H, Hauck RW, H.Worth H, Herth FJF. Position zur Langzeit-Sauerstofftherapie. Pneumologie. 2014; 68: 591-3.

5. Hardinge M, Annandale J, Bourne $S$, et al. British Thoracic Society guidelines for home oxygen use in adults. Thorax. 2015;70:i1-i43.

6. Magnet FS, Schwarz SB, Callegari J, et al. Long-term oxygen therapy: comparison of the German and British guidelines. Respiration. 2017;93:253-63.

7. Magnet FS, Majorski DS, Callegari J, Schwarz SB, Schmoor C, Windisch W, Storre JH. Capillary PO2 does not adequately reflect arterial PO2 in hypoxemic COPD patients. Int J Chron Obstruct Pulmon Dis. 2017;12:2647-53.

8. Struik FM, Sprooten RT, Kerstjens HA, Bladder G, Zijnen M, Asin J, et al. Nocturnal non-invasive ventilation in COPD patients with prolonged hypercapnia after ventilatory support for acute respiratory failure: a randomised, controlled, parallel-group study. Thorax. 2014;69:826-34.

9. Schwarz SB, Callegari J, Hamm C, Windisch W, Magnet FS. Is outpatient control of long-term noninvasive ventilation feasible in COPD patients? Respiration. 2017; in press.

10. Murphy PB, Rehal S, Arbane G, Bourke S, Calverley PMA, Crook AM, et al. Effect of Home Noninvasive Ventilation With Oxygen Therapy vs Oxygen Therapy Alone on Hospital Readmission or Death After an Acute COPD Exacerbation: A Randomized Clinical Trial. JAMA. 2017;317:2177-86. 